\title{
Melatonin rescues impaired penetration ability of human spermatozoa induced by mitochondrial dysfunction
}

\author{
Xue-Ying Zhang, ${ }^{1, *}$, Yi-Meng Xiong,*, Ya-Jing Tan², Li Wang ${ }^{2}$, Rong Li $^{2}$, Yong Zhang², \\ Xin-Mei Liu², Xian-Hua Lin², Li Jin², Yu-Ting Hu², Zhen-Hua Tang², Zheng-Mu Wu², \\ Feng-Hua Yin², Zheng-Quan Wang ${ }^{2}$, Ye Xiao ${ }^{2}$, Jian-Zhong Sheng ${ }^{1,3}$ and He-Feng Huang ${ }^{1,2,4}$ \\ ${ }^{1}$ The Key Laboratory of Reproductive Genetics (Zhejiang University), Ministry of Education, Hangzhou, Zhejiang, \\ China, ${ }^{2}$ The International Peace Maternity and Child Health Hospital, School of Medicine, Shanghai Jiao Tong \\ University, Shanghai, China, ${ }^{3}$ Department of Pathology and Pathophysiology, School of Medicine, Zhejiang \\ University, Hangzhou, Zhejiang, China and ${ }^{4}$ Shanghai Key Laboratory of Embryo Original Diseases, Shanghai, China
}

Correspondence should be addressed to H-F Huang; Email: huanghefg@hotmail.com

*(X-Y Zhang and Y-M Xiong contributed equally to this work)

\begin{abstract}
Fertilization failure often occurs during in vitro fertilization (IVF) cycles despite apparently normal sperm and oocytes. Accumulating evidence suggests that mitochondria play crucial roles in the regulation of sperm function and male fertility. 3-Nitrophthalic acid (3-NPA) can induce oxidative stress in mitochondria, and melatonin, as an antioxidant, can improve mitochondrial function by reducing mitochondrial oxidative stress. The role of sperm mitochondrial dysfunction in fertilization failure during IVF is unclear. The present study revealed that spermatozoa with low, or poor, fertilization rates had swollen mitochondria, increased mitochondriaderived ROS, and attenuated mitochondrial respiratory capacity. 3-NPA treatment enhanced mitochondrial dysfunction in sperm. Spermatozoa with poor fertilization rates, and spermatozoa treated with 3-NPA, had reduced penetration ability. The concentration of melatonin was decreased in semen samples with low and poor fertilization rates. Melatonin, not only decreased excessive mitochondria-derived ROS, but also 'rescued' the reduced penetration capacity of spermatozoa treated with 3-NPA. Taken together, the study suggested that mitochondria-derived ROS and mitochondrial respiratory capacity are independent bio-markers for sperm dysfunction, and melatonin may be useful in improving sperm quality and overall male fertility.

Reproduction (2019) 158 465-475
\end{abstract}

\section{Introduction}

Infertility is defined as the inability to achieve conception after 12 months of regular, unprotected, sexual intercourse, and currently affects approximately $10-15 \%$ of couples around the world (Dyer 2009). Previous studies confirm that $30-40 \%$ of these cases can be explained by male-only factor, $30-40 \%$ by female factors, $10-20 \%$ by combined male and female factors, and another $10-20 \%$ by unexplained factors (Boivin et al. 2007, Gelbaya et al. 2014). In recent years, stress, unhealthy lifestyles and atmospheric pollution have contributed to rising male infertility rates worldwide (Boivin et al. 2007, Dyer 2009, Yang et al. 2017). In vitro fertilization and embryo transfer (IVF-ET) is one of the most effective, assisted reproductive technologies (ARTs) for treatment of these couples (Lutjen et al. 1984, Steer et al. 1992, Van Voorhis 2007). IVF co-cultures spermatozoa with oocytes, which are obtained from both spouses respectively, and creates a fertilized ovum prior to implantation into the uterus
(Lutjen et al. 1984). However, some IVF couples with tubal infertility and normal semen analyses (Tocci \& Lucchini 2010, Barratt et al. 2011) have poor fertilization rates with IVF treatment. The mechanisms underlying these poor fertilization rates are unclear.

Even though the origins and frequency of infertility vary worldwide, 'male factor' infertility contributes to about $50 \%$ of infertile cases overall (Agarwal et al. 2015, Kumar \& Singh 2015). The 'tadpole'-like shape of spermatozoa determines their structural and functional specificity; the head of the spermatozoa includes the acrosome, some cytoplasm, and a nucleus with compacted DNA, the middle-piece contains ordered mitochondria, and the end-piece comprises many longitudinal microfilaments (Stefanini et al. 1967, Egeberg Palme et al. 2018). Abnormalities in cellular structures may cause sperm dysfunction and subsequent male infertility (Ollero et al. 2001, Zhou et al. 2013).

In eukaryotic cells, mitochondria are important for ATP synthesis by oxidative phosphorylation 
(Balaban et al. 2005) and a number of other key processes including ROS generation (Balaban et al. 2005), intracellular calcium recycling and homeostasis (Lim et al. 2015), the intrinsic apoptotic pathways (Green \& Reed 1998), and sex steroid hormones biosynthesis (Stocco 2001). Mitochondria play pivotal roles during spermatogenesis (Rajender et al. 2010), spermatozoa survival and motility (Piomboni et al. 2012), the acrosome reaction and successful fertilization (Gallon et al. 2006), although paternal mitochondria do not play a role in mitochondrial heredity (Luo \& Sun 2013). Any defects in the mitochondrial genome or ultra-structural integrity, as well as abnormalities in mitochondrial membrane potential or energy metabolism, may contribute to impaired sperm function, such as capacitation, the acrosome reaction, oocyte interaction and sperm motility (Amaral et al. 2013). Moreover, increasing evidence indicates that mitochondria-derived ROS is crucial for structural integrity and normal functions of spermatozoa (Clyne 2012). In physiological conditions, spermatozoa generate basal levels of ROS for intracellular signaling activation, capacitation, acrosome reaction and fusion with the oocyte (Clyne 2012). However, uncontrolled and excessive accumulation of ROS in spermatozoa may lead to oxidative stress, which is turn, leads to damage of DNA, or, loss of plasma membrane integrity, or inactivation of enzymes; all of which may further impair sperm function (Tremellen 2008, Clyne 2012).

Melatonin is a hormone that is principally secreted by the pineal gland in a circadian manner in mammals (Manchester et al. 2015), which maintains circadian rhythmicity, regulates aging and reproduction as well as regulating antioxidant activities (Reiter 1980, Manchester et al. 2015, Reiter et al. 2016). Our previous study suggested that melatonin supplementation during diabetic pregnancy improves myocardial ischemic tolerance in diabetic offspring (Gao et al. 2016). Other studies demonstrate that melatonin improves mitochondrial function by reducing mitochondria-derived oxidative stress (Absi et al. 2000, Jimenez-Aranda et al. 2014, Agil et al. 2015, Xu et al. 2016). On the other hand, 3-NPA is an inhibitor of succinate dehydrogenase (SDH) of complex II in the mitochondrial electron transfer chain, which can induce oxidative stress in mitochondria (Cheng et al. 2016).

Because of the crucial roles of mitochondria in sperm function, we propose that mitochondrial dysfunction may be significant in reduced IVF rates. In the present study, we collected sperm specimens from the male partners of 400 infertile couples. The semen parameters of these male partners were normal; however, the in vitro fertilization (IVF) rates of oocytes inseminated by these sperm were different. We examined possible associations between sperm mitochondria status and IVF rates. Furthermore, we explored the potential mechanisms as to how mitochondrial dysfunction impairs fertilization of human sperm, and whether treatment with melatonin might 'rescue' any such impairment.

\section{Materials and methods}

\section{Media and reagents}

We used the standard, human spermatozoa culture media throughout the present studies (IVF-PLUS and G-MOPS, Vitrolife, Gothenburg, VastraGotaland, Sweden) and a spermatozoa preparation medium for purification (ISolate Irvine Scientific, Santa Ana, CA, USA). IVF-PLUS is a bicarbonate buffered medium containing human serum albumin, and G-MOPS is a MOPS buffered medium free of protein. 3-nitropropionic (3-NPA) and melatonin were provided by Sigma-Aldrich. Mitotracker Green FM, MitoSOX and wheat germ agglutinin (WGA) were obtained from Invitrogen. Human melatonin ELISA kits were provided by IBL international (Flughafenstr, Hamburg, Germany).

\section{Samples collection}

Human semen samples were obtained from the male partners of 400 infertile couples in the reproductive center at International Peace Maternity and Child Health Hospital, Shanghai, China. These patients underwent IVF-ET. Among 400 infertile couples, 390 couples $(97.5 \%)$ had tubal infertility in the female partners and 10 couples $(2.5 \%)$ were diagnosed as unexplained infertility. Male partners with abnormal semen parameters, or female partners with polycystic ovary syndrome (PCOS), were excluded (Rotterdam ESHRE/ASRM-Sponsored PCOS Consensus Workshop Group 2004). Based on IVF rates (FR, the ratio of oocytes fertilized to oocytes inseminated in IVF), the infertile couples were divided into three groups: FR1 (FR $>65 \%, n=299)$, FR2 (FR $>30-\leq 65 \%, n=79)$ and FR3 (FR $\leq 30 \%, n=22$ ).

\section{Ethical approval}

The study was approved by the ethics committee on human research at International Peace Maternity and Child Health Hospital, Shanghai, China. All patients provided written informed consent. All animal experiments were approved by the Institute's Animal Ethics Committee of Shanghai Jiao Tong University, and were performed in accordance with the guidelines on the use of laboratory animals.

\section{Human spermatozoa preparation and culture}

Human sperm were obtained from freshly ejaculated semen from male partners with normal, low and poor IVF rates, respectively. After 3 days of abstinence, morphology and motility were assessed by Papanicolaou and computer-assisted sperm analyzer (CASA) (HTM-IVOS system, version 12, Hamiltom-Thorne Research, Beverly, CA, USA), respectively according to WHO standards. Semen samples were liquefied at $37^{\circ} \mathrm{C}$ in a shaker for $30 \mathrm{~min}$ and purified using Isolate (Irvine Scientific) by discontinuous density centrifugation. A twolayer gradient was prepared by using 'ready-to-use' solutions 
of upper and lower isolate (Irvine Scientific). Semen samples were placed on top of the upper layer in a $15 \mathrm{~mL}$ centrifuge tube and centrifuged at $254 \mathrm{~g}$ for $20 \mathrm{~min}$. Pellets of motile sperm at the bottom of the lower isolate (Irvine Scientific) were aspirated and washed once with IVF-PLUS (Vitrolife). Purified sperm were incubated in IVF-PLUS (Vitrolife) at concentrations of $3 \times 10^{7}$ / $\mathrm{mL}$ with or without treatment with different concentrations of 3-NPA (0, 0.5 or $1 \mathrm{mM}$ ) (Rosenstock et al. 2004, Perez-De La Cruz et al. 2006) and/or melatonin and incubated in one cell culture incubator (Thermo Fisher Scientific) for $3 \mathrm{~h}$ before further investigation. For melatonin treatment, melatonin (1 M) in sterile anhydrous DMSO solution was diluted with IVF-PLUS (Vitrolife) to a final concentration of $0.3 \mathrm{mM}$ or $0.5 \mathrm{mM}$, and sperm were cultured in IVF-PLUS (Vitrolife) containing melatonin for $3 \mathrm{~h}$ before further assays. Treated sperm were used to analyze the roles of 3-NPA and melatonin in mitochondrial respiratory capacity, mitochondria-derived ROS, kinematic parameters and penetration ability of sperm.

\section{Measurement of kinematic parameters of sperm}

Sperm motility, progressive motility and other kinematic parameters were assayed by CASA (HTM-IVOS system, version 12, Hamiltom-Thorne Research, Beverly, CA, USA) including straight line velocity $(\mathrm{VSL}, \mu \mathrm{m} / \mathrm{s})$, curvilinear velocity $(\mathrm{VCL}$, $\mu \mathrm{m} / \mathrm{s}$ ), average path velocity (VAP), amplitude of lateral head displacement $(\mathrm{ALH}, \mu \mathrm{m})$, straightness of trajectory $(\mathrm{STR}, \%)$ expressed as VSL/VCL, linearity of trajectory (LIN,\%) expressed as VSL/VAP, beat cross frequency $(\mathrm{BCF}, \mathrm{Hz})$ and wobble of trajectory (WOB, \%) expressed as VAP/VCL. The settings of CASA parameters were listed in Supplementary Table 1 (see section on supplementary data given at the end of this article). Sperm suspension $(3 \mu \mathrm{L})$ was added to a four-chamber slide (Leja, Nieuw-Vennep, Haarlemmermeer, Netherlands) for analysis. Four different fields per ejaculate containing 200300 sperm were analyzed. Triplicate determinations for each ejaculate were performed. All measurements of spermatozoa samples were performed at $37^{\circ} \mathrm{C}$.

\section{Measurement of mitochondrial mass}

Purified human sperm cultured in IVF-PLUS (Vitrolife) were harvested by centrifugation and washed twice with Hanks buffer. $2 \times 10^{6}$ spermatozoa were incubated in $200 \mu \mathrm{L}$ Hanks buffer containing $200 \mathrm{nM}$ Mitotracker Green FM (CottetRousselle et al. 2011, Yamamori et al. 2012) and probed at $37^{\circ} \mathrm{C}$ for $20 \mathrm{~min}$ in dark. Then, spermatozoa were pelleted by centrifugation at $800 \mathrm{~g}$ for $5 \mathrm{~min}$ at room temperature and washed three times with warm Hanks buffer. Mitochondrial mass (Cottet-Rousselle et al. 2011, Yamamori et al. 2012) of spermatozoa was analyzed by flow cytometry (Beckman Coulter) using $419 \mathrm{~nm}$ excitation laser and $516 \mathrm{~nm}$ emission laser, respectively. 7-Amino-Actinomycin D (7-AAD) was used to exclude dead sperm. In single, alive sperm population, the mean fluorescence intensity (MFI) of Mitotracker Green FM was used to quantify mitochondrial mass in sperm. Flowjo 10.0 software was used to analyze the data of flow cytometry.

\section{Measurement of mitochondrial ROS}

Mitochondria-derived ROS in spermatozoa was studied with mitochondrial superoxide indicator, MitoSOX (CottetRousselle et al. 2011, Yamamori et al. 2012). Purified human sperm cultured in IVF-PLUS (Vitrolife) were pelleted by centrifugation and washed twice with warm Hanks buffer. Then, $2 \times 10^{6}$ spermatozoa were resuspended with $200 \mu \mathrm{L}$ Hanks buffer containing $3 \mu \mathrm{M}$ MitoSOX and incubated at $37^{\circ} \mathrm{C}$ for 15 min in the dark. Spermatozoa were gently washed three times with warm Hanks buffer by centrifugation at $800 \mathrm{~g}$ for 5 min. Flow cytometry (Beckman Coulter) and fluorescence microscopy (Zeiss) were used to evaluate mitochondrial ROS in spermatozoa at excitation and emission of approximately $510 \mathrm{~nm}$ and $580 \mathrm{~nm}$. 7-AAD was used to exclude dead sperm. In the population of alive sperm, the MFI of MitoSOX was used to quantify mitochondrial ROS in sperm. FlowJo 10.0 software was used to analyze the data of flow cytometry.

\section{Transmission electron microscopy}

Purified human spermatozoa cultured in IVF-PLUS (Vitrolife) were collected by centrifugation, and then $1 \times 10^{7}$ sperm were fixed with $2.5 \%(\mathrm{v} / \mathrm{v})$ glutaraldehyde in phosphate buffered saline (PBS, 0.1 M, pH 7.0) overnight. Spermatozoa were embedded in liquid agarose and solidified for $15 \mathrm{~min}$ at $4{ }^{\circ} \mathrm{C}$, before being cut into small portions and post-fixed in $1 \%$ osmium tetroride $\left(\mathrm{O}_{\mathrm{s}} \mathrm{O}_{4}\right)$ for $1 \mathrm{~h}$ and washed again three times in PBS $(0.1 \mathrm{M}, \mathrm{pH} 7.0)$. After being dehydrated through a graded series of ethanol, the samples were transferred into absolute acetone for $20 \mathrm{~min}$. Sperm samples were embedded in spur resin and heated at $70^{\circ} \mathrm{C}$ for more than $9 \mathrm{~h}$, and then sectioned with LEICA EM UC7 ultratome before staining with uranyl acetate and then alkaline lead citrate for 5 and $10 \mathrm{~min}$, respectively. The sections were imaged with Hitachi Code H7650 TEM (Hitachi). Four samples of spermatozoa in each group (FR1, 2 and 3), and 20-40 sperm in each sample, were observed and analyzed.

\section{Measurement of mitochondrial respiratory capacity in live sperm by seahorse XF cell mito stress test system}

Seahorse XF cell mito stress test system was adopted to analyze mitochondrial respiratory capacity (Pelgrom et al. 2016, Van Der Windt et al. 2016). Purified sperm cultured in IVF-PLUS (Vitrolife) were collected by centrifugation, and washed twice in non-carbonate buffered G-MOPS medium (Vitrolife), and resuspended in G-MOPS medium to a density of $3.0 \times 10^{7} /$ $\mathrm{mL}$. The sperm suspension $(160 \mu \mathrm{L})$ was seeded in each well of a seahorse XF96 cell culture microplate and incubated in the non- $\mathrm{CO}_{2} 37^{\circ} \mathrm{C}$ cell incubator for $3 \mathrm{~h}$. During this period, the seahorse sensor cartridge was loaded with $20 \mu \mathrm{L}$ of $18 \mu \mathrm{M}$ oligomycin (Penefsky 1985), $10 \mu \mathrm{M}$ FCCP (Benz \& Mclaughlin 1983), and a mixture of $11 \mu \mathrm{M}$ rotenone ( $\mathrm{Li}$ et al. 2003) and $11 \mu \mathrm{M}$ antimycin A (Lai et al. 2005), respectively. The final concentrations in each well of sperm suspension were $2 \mu \mathrm{M}$ oligomycin, $1 \mu \mathrm{M}$ FCCP, $1 \mu \mathrm{M}$ rotenone and antimycin $\mathrm{A}$ mixture. Seahorse assay was undertaken by monitoring oxygen 
consumption rate (OCR) per well of human spermatozoa. Oligomycin, FCCP, and a mixture of rotenone and antimycin A were serially injected into each well of human sperm suspension to measure ATP-linked respiration, maximal respiration and non-mitochondrial respiration, respectively (Pelgrom et al. 2016, Van Der Windt et al. 2016). The Seahorse Cell Mito Stress Test Report Generator was used to analyze mitochondrial respiratory capacity.

\section{Detection of melatonin in semen plasma samples}

After liquefying at $37^{\circ} \mathrm{C}$ for $30 \mathrm{~min}$, human semen samples were centrifuged at $3500 \mathrm{~g}$ for $10 \mathrm{~min}$. Seminal plasma samples were obtained from the centrifuged supernatant of liquefied semen. Then melatonin concentration in seminal plasma was determined using ELISA Kit (RE54021, IBL international) according to the manufacturer's instructions.

\section{Human spermatozoa penetration of hamster zona- free oocytes}

Female, seven-week-old hamsters weighing approximately $110 \mathrm{~g}$ were superovulated by intraperitoneal (i.p.) injection of $30 \mathrm{IU}$ pregnant mare's serum gonadotropin (Lapolt et al. 1992) in $100 \mu \mathrm{L}$ saline solution $(0.9 \% \mathrm{NaCl})$. Fifty-four hours later, each female hamster received an i.p. injection of $40 \mathrm{IU}$ of hCG in $100 \mu \mathrm{L}$ saline solution $(0.9 \% \mathrm{NaCl})$. Seventeen hours later, the hamsters were anesthetized and killed by i.p. injection of $3 \mathrm{~mL}$ $0.5 \%(\mathrm{w} / \mathrm{v})$ pentobarbital sodium in $0.9 \% \mathrm{NaCl}$. Oviducts were excised and placed in ice-cold Hanks buffer. Ova enclosed by cumulus were expelled from the bulbous section of the duct with 26 gauge needle and transferred into $2 \mathrm{~mL}$ GAMET (Vitrolife) containing $8 \mathrm{IU} / \mathrm{mL}$ hyaluronidase (Vitrolife) for $3 \mathrm{~min}$ at $37^{\circ} \mathrm{C}$ to remove cumulus mass enclosing ova. The ova were washed three times with GAMET (Vitrolife). Zona pellucida was then removed by transferring zona intact ova into GAMET (Vitrolife) containing $1 \mathrm{mg} / \mathrm{mL}$ trypsin (Sigma) for $2 \mathrm{~min}$. The zona-free ova were then washed three times in GAMET (Vitrolife) and transferred into $90 \mu \mathrm{L}$ IVF-PLUS (Vitrolife) drops covered by mineral oil (Vitrolife). Human sperm suspension $(10 \mu \mathrm{L})$ with motile sperm concentration of $3.0 \times 10^{7} / \mathrm{mL}$ was added into the droplet containing ova (10 ova per droplet) to arrive at a final concentration of $3.0 \times 10^{6}$ human sperm per milliliter. After 8-10h, ova were washed twice with IVF-PLUS (Vitrolife) and stained with WGA (Life technology) and DAPI (Vectashield). The number of penetrated sperm per ovum was recorded by confocal imaging method, and the sperm penetration index was the average number of penetrated sperm per ovum.

\section{Statistical analysis}

All experiments were repeated at least three times with triplicate determination. Data were analyzed using SPSS 16.0 for Windows (SPSS, Inc). The Student $t$ test was used to evaluate statistical significance between two groups. One-way analysis of variance (ANOVA) with the Tukey's post hoc tests was used to evaluate the statistical significance of the difference between more than two groups. $P$ value $<0.05$ was considered statistically significant.

\section{Results}

\section{Basal clinical characteristics and routine semen analysis of unexplained infertile patients}

In this study, we examined the semen parameters of males from 400 infertile couples and found that all parameters were in the normal range of reference values defined by WHO fifth edition (Table 1). Compared to FR1 group, the percentages of normal morphology, progressive motility and ALH of sperm in FR2 group were slightly reduced $(8.6 \pm 3.8 \%$ vs $10.1 \pm 4.2 \%$, $51.3 \pm 10.9 \%$ vs $54.6 \pm 11.2 \%$ and $4.8 \pm 1.3$ vs $5.1 \pm 1.2$, respectively, $P<0.05$ ) (Table 1 ). However, the average IVF rates in FR2 and FR3 groups were $51.3 \pm 10.9 \%$ and $7.2 \pm 9.5 \%$, respectively, which were much lower than those in FR1 group $(87.7 \pm 10.9 \%)$. Because the cause of infertility in these males could not be explained by the parameters from routine semen analysis, we called these 'unexplained' infertile males. Strikingly, after treatment with ICSI in a subsequent cycle with a new oocytes retrieval, the average fertilization rate in FR3 group reached $82.8 \pm 15.5 \%$ (Table 1$)$. The results suggest that reduced penetration ability of sperm may be important in these 'unexplained' infertile males.

\section{Impaired ultra-structures and increased $m R O S$ were observed in mitochondria of sperm with low or poor IVF rates}

To identify whether mitochondrial impairment was involved in sperm dysfunction of unexplained infertile males, firstly we analyzed mitochondrial structures of sperm with transmission electric microscopy (TEM). The data showed that swelling and metamorphosing mitochondria were widely detected in sperm from FR2 and FR3 groups (Fig. 1A). Vacuolation and reduced cristae were also extensively distributed in mitochondria of these sperm from FR2 and FR3 groups (Fig. 1A). We also examined mROS in sperm using MitoSOX staining and found that, compared to FR1 group, the MFI of MitoSOX was significantly higher in mitochondria of sperm from FR2 and FR3 groups than that from FR1 group, which indicated that mROS was much increased in sperm from FR2 and FR3 groups compared to that from FR1 group (Fig. 1B and C). However, there was no significant difference in mitochondrial mass in sperm among groups of FR1, FR2 and FR3 (Fig. 1D).

\section{Mitochondrial respiratory capacity was inhibited in sperm with low, or poor, IVF rates}

In this study, mitochondrial respiratory capacity was analyzed using the seahorse system. The data showed that mitochondrial respiratory capacity of sperm in FR2 and FR3 groups were significantly reduced compared to that in FR1 group (Fig. 2A). We also found that basal OCR, oligomycin-induced OCR, FCCP-induced OCR as 
Table 1 Basal clinical characteristics of infertile couples and routine semen analysis.

\begin{tabular}{|c|c|c|c|}
\hline Characteristics & FR1 (>65\%) & FR2 $(>30-\leq 65 \%)$ & FR3 $(\leq 30 \%)$ \\
\hline$n$ & 299 & 79 & 22 \\
\hline Primary infertility, $n(\%)$ & $146(48.8)$ & $45(57.0)$ & $11(50.0)$ \\
\hline Secondary infertility, $n(\%)$ & $153(51.2)$ & $34(43.0)$ & $11(50.0)$ \\
\hline Infertility duration, years & $3.8 \pm 2.9(1-20)$ & $3.8 \pm 2.4(1-13)$ & $4.3 \pm 2.3(1-9)$ \\
\hline Tubal infertility, $n(\%)$ & $291(97.3)$ & $78(98.7)$ & $21(95.5)$ \\
\hline Unexplained infertility, $n(\%)$ & $8(2.7)$ & $1(1.3)$ & $1(4.5)$ \\
\hline No. of oocytes inseminated & $10.8 \pm 5.3(3-28)$ & $10.6 \pm 4.7(3-24)$ & $13.4 \pm 7.4(3-30)$ \\
\hline No. of oocytes fertilized & $9.4 \pm 4.6(2-26)$ & $5.5 \pm 2.7(1-14)$ & $1.1 \pm 1.8(0-7)$ \\
\hline FR after IVF, \% & $87.7 \pm 10.9(66.7-100)$ & $51.3 \pm 10.9(31.3-65.0)$ & $7.2 \pm 9.5(0-28.6)$ \\
\hline FR after ICSI, \% & -1 & - & $82.8 \pm 15.5(50.0-100)$ \\
\hline \multicolumn{4}{|l|}{ Data on females } \\
\hline Age, years & $32 \pm 4(21-43)$ & $31 \pm 4(22-40)$ & $32 \pm 4(25-39)$ \\
\hline Menarche, years & $13.8 \pm 1.1(11-18)$ & $13.6 \pm 1.1(12-18)$ & $14.1 \pm 0.8(12-16)$ \\
\hline Menstrual period, days & $30.2 \pm 3.2(23-40)$ & $29.8 \pm 3.1(22-40)$ & $30.2 \pm 3.2(25-40)$ \\
\hline Abortion history, $n(\%)$ & $133(44.5)$ & $27(34.2)$ & $10(45.5)$ \\
\hline \multicolumn{4}{|l|}{ Data on males } \\
\hline Age, years & $33 \pm 5(23-50)$ & $32 \pm 4(25-43)$ & $34 \pm 3(27-39)$ \\
\hline \multicolumn{4}{|l|}{ Semen analysis } \\
\hline$\%$ of normal morphology of sperms & $10.1 \pm 4.2(4.0-26.0)$ & $8.5 \pm 3.7(4.0-23.0)^{* *}$ & $9.8 \pm 3.1(4.0-16.0)$ \\
\hline Sperm concentration, $10^{6} / \mathrm{L}$ & $61.3 \pm 37.5(15.1-236.2)$ & $53.9 \pm 37.9(15.2-295.9)$ & $59.0 \pm 44.9(19.9-242.0)$ \\
\hline Progressive motile sperms, \% & $54.6 \pm 11.2(32.4-82.9)$ & $51.2 \pm 11.4(32.3-79.7)^{*}$ & $53.4 \pm 11.9(33.8-78.2)$ \\
\hline Motile sperms, \% & $65.4 \pm 11.2(42.3-94.1)$ & $62.9 \pm 12.3(42.9-87.8)$ & $62.2 \pm 12.7(40.0-86.0)$ \\
\hline $\mathrm{VCL}, \mathrm{um} / \mathrm{s}$ & $96.9 \pm 20.4(47.3-160.6)$ & $92.2 \pm 20.0(50.5-146.4)$ & $95.9 \pm 15.3(64.0-121.0)$ \\
\hline VSL, um/s & $49.5 \pm 11.2(20.0-88.1)$ & $48.0 \pm 12.2(24.1-84.2)$ & $55.6 \pm 9.1(34.1-68.4)$ \\
\hline VAP, um/sec & $60.3 \pm 11.2(32.0-101.2)$ & $58.0 \pm 11.7(33.2-91.1)$ & $60.6 \pm 8.8(43.8-78.0)$ \\
\hline LIN, \% & $52.4 \pm 8.1(33.7-70.5)$ & $53.3 \pm 8.9(31.5-69.6)$ & $53.7 \pm 5.9(44.6-62.7)$ \\
\hline ALH, um & $5.1 \pm 1.2(2.3-8.8)$ & $4.8 \pm 1.2(2.4-8.2)^{*}$ & $4.9 \pm 0.9(3.2-6.2)$ \\
\hline STR, \% & $79.7 \pm 6.8(58.5-91.3)$ & $80.0 \pm 7.5(60.7-91.3)$ & $81.4 \pm 5.3(69.9-89.2)$ \\
\hline $\mathrm{BCF}, \mathrm{Hz}$ & $24.2 \pm 3.3(16.5-34.1)$ & $24.7 \pm 4.2(16.4-35.2)$ & $24.3 \pm 3.3(17.7-30.4)$ \\
\hline WOB, \% & $63.8 \pm 5.4(50.7-84.8)$ & $64.5 \pm 5.6(51.4-74.9)$ & $64.2 \pm 3.6(58.8-71.2)$ \\
\hline $\mathrm{BMI}\left(\mathrm{kg} / \mathrm{m}^{2}\right)$ & $24.6 \pm 3.6(16.0-39.2)$ & $24.4 \pm 3.4(19.2-35.9)$ & $24.0 \pm 3.1(17.3-30.1)$ \\
\hline \multicolumn{4}{|l|}{ BMI category, n (\%) } \\
\hline Underweight: $<18.5$ & $8(2.7)$ & $0(0)$ & $1(4.5)$ \\
\hline Normal weight: $18.5-24.9$ & $157(52.5)$ & $41(51.9)$ & $12(54.5)$ \\
\hline Overweight: 25.0-29.9 & $108(36.1)$ & $33(41.8)$ & $8(36.4)$ \\
\hline Obese: $\geq 30.00$ & $26(8.7)$ & $5(6.3)$ & $1(4.5)$ \\
\hline
\end{tabular}

Data $=$ mean \pm S.E.M.

* and $* * P<0.05$ and $P<0.01$, respectively, compared to FR1 group.

well as rotenone plus antimycin-induced OCR of sperm from males in FR2 and FR3 groups were significantly lower than that in FR1 group, as indicated by an evident impairment of mitochondrial respiratory capacity (Fig. 2B). Furthermore, the significant decrement of FFCPinduced OCR also suggested a reduction of mitochondrial reserve respiratory capacity in sperm from unexplained infertile males in FR2 and FR3 groups (Fig. 2B).

\section{3-NPA treatment reduced mitochondrial respiratory capacity and the penetration capacity of sperm from infertile males of FR1 group}

In order to analyze the impact of 3-NPA on sperm mitochondria, mitochondrial respiratory capacity and mitochondria-derived ROS were determined by seahorse system and MitoSOX staining. Compared to the control (0 mM 3-NPA treatment), treatment of sperm with 0.5 and $1 \mathrm{mM} 3-\mathrm{NPA}$ significantly attenuated the mitochondrial respiratory capacity $(P<0.05$, Fig. 3A). Basal, oligomycin-induced, FCCP-induced, and rotenone plus antimycin-induced OCR were significantly lower in sperm treated with $0.5 \mathrm{mM}$ and $1 \mathrm{mM} 3-\mathrm{NPA}$, respectively, than controls $(P<0.05$, Fig. $3 \mathrm{~B})$. The patterns of reduced mitochondrial respiratory capacity of sperm treated with $0.5 \mathrm{mM}$ and $1 \mathrm{mM}$ 3-NPA, respectively, were similar to those of sperm in the FR2 or FR3 group (Fig. 2A and B). 3-NPA treatment also significantly increased MitoSOX staining intensities $(P<0.05$, Fig. 3C), indicating excessive ROS production in the mitochondria. We evaluated kinematic parameters of sperm treated with 0.5 and $1 \mathrm{mM} 3-\mathrm{NPA}$, respectively, and found that there was no significant difference in the parameters including motility, VCL, VSL, VAP, LIN, STR, BCF and WOB between sperm treated with or without 3-NPA (Fig. 3D, E, F, G, H, I, J and K). However, progressive motility and ALH were slightly depressed in the sperm treated with $1 \mathrm{mM} 3-\mathrm{NPA}$ (Fig. $3 \mathrm{~L}$ and M). 
A
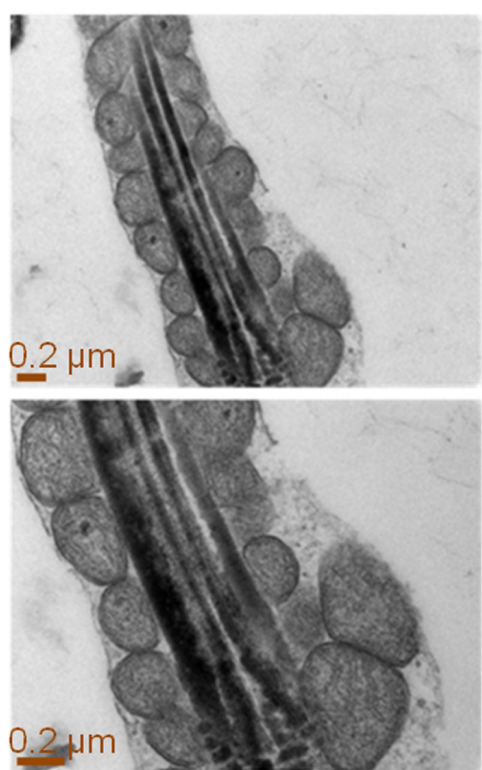

B

FR1

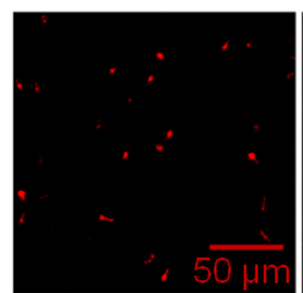

FR2
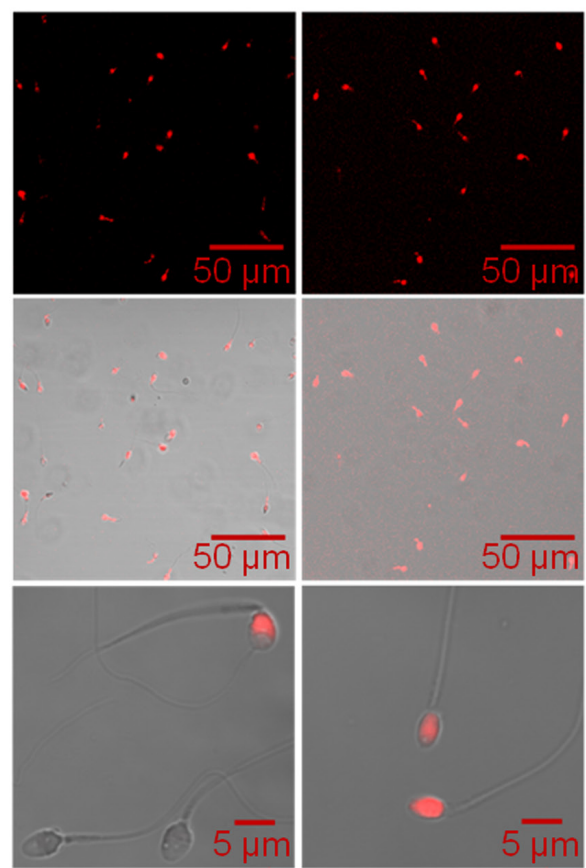

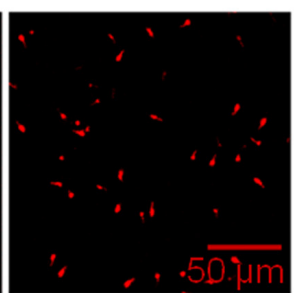

C
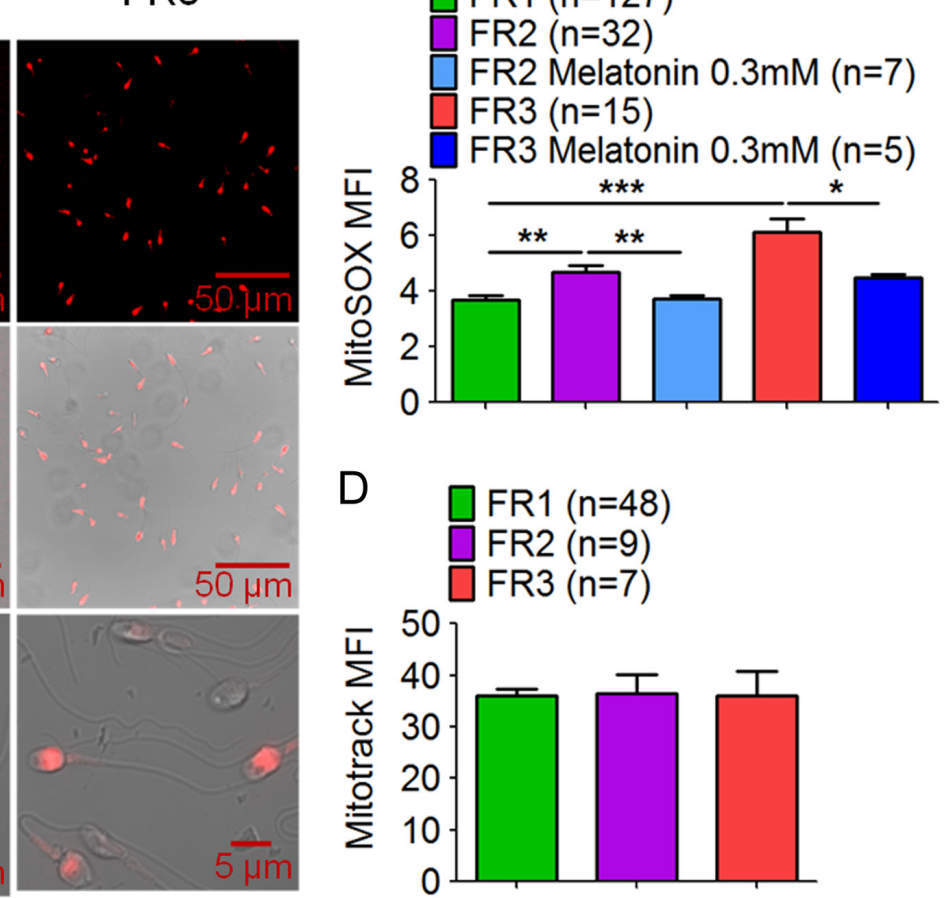

Figure 1 Impaired mitochondrial ultra-structure and increased mitochondria-derived ROS are observed in sperm of unexplained infertile male patients. (A) Mitochondrial ultra-structures in sperm from FR1, FR2 and FR3 groups were observed by TEM. The red arrows indicate impaired mitochondrial ultra-structure (such as metamorphism, black bulk, physalides and decreased cristae) in sperm from group FR2 and FR3. Four samples in each group (FR1, 2 and 3), and 20-40 sperm in each sample, were analyzed. Scale bar $=0.2 \mu \mathrm{m}$. (B) The distribution and content of mitochondria-derived ROS in sperm from FR1, FR2 and FR3 groups were analyzed by MitoSOX staining and fluorescence microscopy. Red color indicates MitoSOX staining. The middle and lower panels showed the overlapped pictures of fluorescence and bright fields. Scale bar $=50 \mu \mathrm{m}$ in the upper and middle panels, scale bar $=5 \mu \mathrm{m}$ in the lower panel. (C) The MFI of MitoSOX staining in sperm from FR1, FR2 and FR3 groups in the absence or presence of $0.3 \mathrm{mM}$ melatonin was assessed by flow cytometry and shown as bar graph (mean \pm S.E.M.). *, ** and ***, $P<0.05, P<0.01$ and $P<0.001$, respectively, determined by ANOVA with the Tukey's post hoc test. Data are representative of three independent experiments. (D) Mitochondrial mass in sperm was assessed by Mitotracker Green FM staining and flow cytometry and shown as bar graph (mean \pm S.E.M.). $n$, number of samples. Data are representative of three independent experiments. 

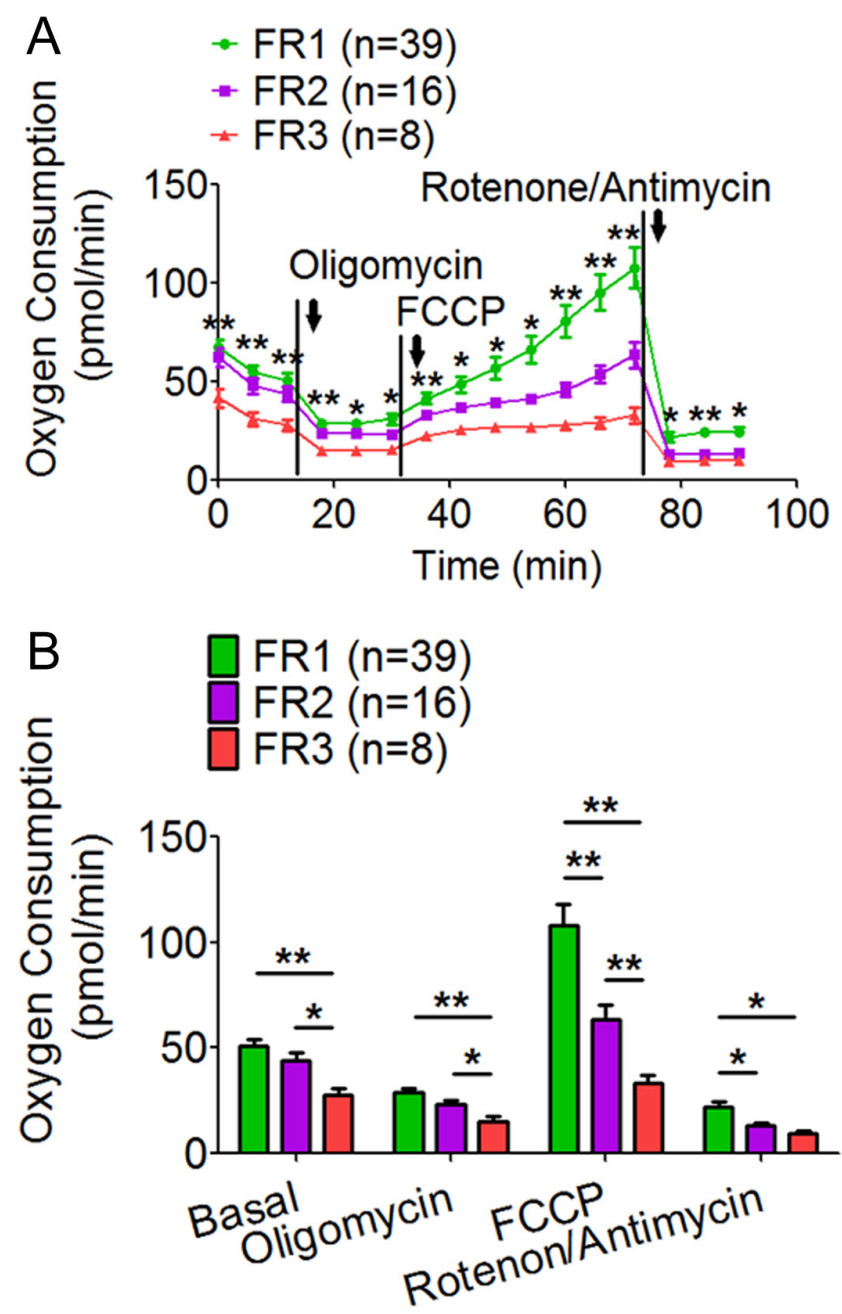

Figure 2 Mitochondrial respiratory capacity is reduced in sperm of FR2 and FR3 groups compared to that of FR1 group. (A) Mitochondrial respiratory curves of sperm were assessed with Seahorse system. ${ }^{\prime * \prime}$ or ${ }^{* * \prime}$ indicates significant difference when compared FR3 with FR1, $P$ values were determined by the Student $t$ test. (B) Basal OCR, oligomycin-induced OCR, FCCP-induced OCR and rotenone plus antimycin-induced OCR of mitochondria in sperm from FR1, FR2 and FR3 groups were shown as bar graph

(mean \pm S.E.M.). $P$ values were determined by ANOVA with the Tukey's post hoc test. ${ }^{*}$ and ${ }^{* *}, P<0.05$ and $P<0.01$, respectively. $n$, number of samples. Data are representative of three independent experiments.

In order to confirm whether oxidative stress could lead to fertilization failure, we evaluated the fertilization potential of the sperm treated with, or without, 3-NPA by determining penetration ability of sperm into hamster zona-free oocytes. Sperm with normal fertilization rate were collected from the normal men in FR1 group. After treatment of sperm with $1 \mathrm{mM} 3-\mathrm{NPA}$, the penetration ability of sperm into hamster zona-free oocytes was reduced by $50 \%$ compared to $0 \mathrm{mM} 3-\mathrm{NPA}$ treatment (Fig. 4A and B).

\section{Melatonin treatment rescues mitochondrial oxidative stress and improves kinematic parameters as well as the penetration ability of sperm}

The role of melatonin in sperm and male infertility is undefined. In the study, the concentrations of melatonin in semen plasma samples of infertile males from three groups were determined by ELISA. The data showed that the concentrations of melatonin in semen samples from FR2 and FR3 groups were evidently decreased compared to that of FR1 (Fig. 4A), which suggested that melatonin might play a significant role in unexplained infertility. First, we considered whether melatonin has an antioxidant effect in sperm; our data show that melatonin treatment reversed abnormal increases of mitochondriaderived ROS in spermatozoa from FR2 and FR3 groups (Fig. 1C) as well as 3-NPA-treated sperm $(P<0.05$, Fig. $3 \mathrm{C})$. Second, treatment of sperm with $0.3 \mathrm{mM}$ melatonin significantly improved not only progressive motility and $\mathrm{ALH}$, but also penetration ability of sperm into hamster zona-free oocytes in the presence of $1 \mathrm{mM} 3-\mathrm{NPA}$ (Figs $3 \mathrm{~L}$ and $\mathrm{M}, 4 \mathrm{~B}$ and $\mathrm{C}$ ). Finally, we evaluated the role of melatonin in sperm penetration ability from unexplained infertile males with poor FR. Strikingly, following treatment of sperm from unexplained infertile males in FR3 group with 0.3 and $0.5 \mathrm{mM}$ melatonin, respectively, the penetration index of sperm into hamster zona-free oocytes was increased from $1.2 \pm 0.3$ (control, without melatonin treatment) to $4.0 \pm 0.8(0.3 \mathrm{mM}$ melatonin treatment) and $7.3 \pm 0.6(0.5 \mathrm{mM}$ melatonin treatment), respectively (Fig. 4D and E).

\section{Discussion}

Although the development of assisted reproduction technologies has helped millions of infertile couples have their own babies (Lutjen et al. 1984, Van Voorhis 2007), the etiology of male infertility during natural reproduction or assisted reproduction is still not fully understood. In this study, we focused on one pattern of atypical, male infertility, that we termed 'unexplained' male infertility, and explored its possible pathogenesis. Our clinical investigation demonstrated that 20\% (79/400) infertile couples had low IVF rate $(51.3 \pm 10.9 \%)$ and $6 \%(22 / 400)$ infertile couples had poor IVF rate (7.2 $\pm 9.5 \%)$. Strikingly, the clinical investigation found that ICSI (intracytoplasmic sperm injection), an IVF procedure in which a single sperm cell is injected directly into the cytoplasm of an egg, could dramatically improve IVF rates in infertile couples from 7.2 to $82.8 \%$. These data suggested that reduced penetration ability of spermatozoa probably contributes to decreased IVF rates in these infertile couples undergoing conventional IVF-ET, which could not be detected by routine semen analysis.

Mitochondria play essential roles in the regulation of sperm functions (Rajender et al. 2010, Amaral et al. 2013). 

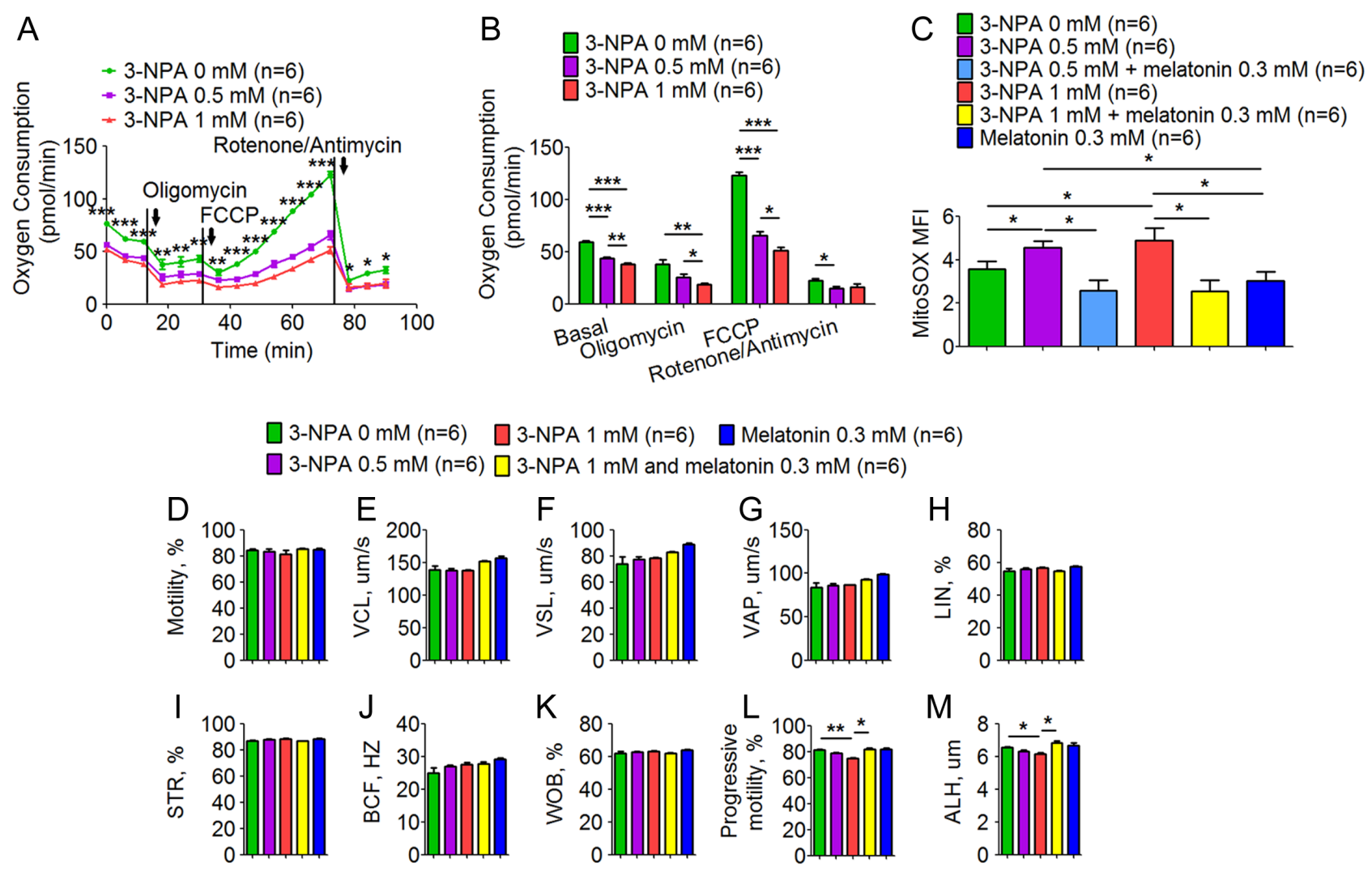

Figure 3 Effects of 3-NPA on mitochondrial respiratory capacity, mitochondria-derived ROS and kinematic parameters of sperm with normal IVF rate from FR1 group. (A) Mitochondrial respiratory curves were assessed by Seahorse system for sperm treated without or with 3-NPA (0.5 mM or $1 \mathrm{mM})$, respectively. ${ }^{\prime * \prime},{ }^{\prime * * \prime}$ or ${ }^{\prime * * * \prime}$ indicates significant differences when compared 3-NPA $1 \mathrm{mM}$ group with 3-NPA $0 \mathrm{mM}$ group. $P$ values were determined by the Student $t$ test. (B) Basal OCR, oligomycin-induced OCR, FCCP-induced OCR and rotenone plus antimycin-induced OCR of sperm in the absence or presence of 3-NPA $(0.5 \mathrm{mM}$ or $1 \mathrm{mM})$ were shown as bar graph (mean \pm S.E.M.). $P$ values were determined by ANOVA with the Tukey's post hoc test. (C) The MFI of MitoSOX staining in sperm treated without or with 3-NPA $(0.5 \mathrm{mM}$ or $1 \mathrm{mM})$ in the absence or presence of $0.3 \mathrm{mM}$ melatonin was assessed by flow cytometry and shown as bar graph (mean \pm S.E.M.). $P$ values were determined by ANOVA with the Tukey's post hoc test. (D, E, F, G, H, I, J, K, L and M) The kinematic parameters of sperm treated without or with 3-NPA (0.5 mM or $1 \mathrm{mM}$ ) in the absence or presence of $0.3 \mathrm{mM}$ melatonin were assessed by CASA and shown as bar graph (mean \pm S.E.M.). $P$ values were determined by ANOVA with the Tukey's post hoc test. ${ }^{*}, * *$ and $* * * P<0.05, P<0.01$ and $P<0.001$, respectively. $n$, number of samples. Data are representative of three independent experiments.

In this study, we examined the mitochondrial status of sperm from males with 'unexplained' infertility with several strategies. We found that the ultra-structures of sperm mitochondria from these unexplained infertile males were impaired. Moreover, mitochondria-derived oxidative stress was increased, while the mitochondrial respiratory capacity was reduced in their sperm, which indicated that dysfunctional mitochondria probably led to this type of unexplained male infertility. Previous studies have shown that mitochondrial dysfunction might impair the motility of sperm (Piomboni et al. 2012, Amaral et al. 2013). However, we did not find significantly impaired mobility in sperm of unexplained infertile males. Overall, our results indicate that although sperm with poor IVF rates looked normal in the routine semen analysis, their mitochondria might be impaired and dysfunctional.

3-NPA is an inhibitor of succinate dehydrogenase (SDH) of complex II in mitochondrial electron transfer chain (Cheng et al. 2016), and 3-NPA treatment induces oxidative stress in mitochondria and leads to mitochondrial dysfunction (Cheng et al. 2016). In this study, we treated normal sperm with 3-NPA to simulate impaired mitochondria in sperm of unexplained infertile males. We found that 3-NPA had little impact on the parameters of routine analysis; however, 3-NPA dramatically decreased oxygen consumption and increased the production of mitochondria-derived ROS in sperm. These results were consistent with our clinical investigations and suggested that impaired mitochondrial respiratory capacity and increased ROS might play important roles in sperm with poor IVF rate from 'unexplained' infertile males.

The core strategy of IVF is to co-culture sperm with oocytes in vitro to produce a fertilized ovum (Lutjen et al. 1984). Sperm have enough chances to meet oocytes during IVF manipulation. However, poor IVF rates were observed in some patients and led to IVF failure. ICSI can be used to improve fertilization rates in some cases. 

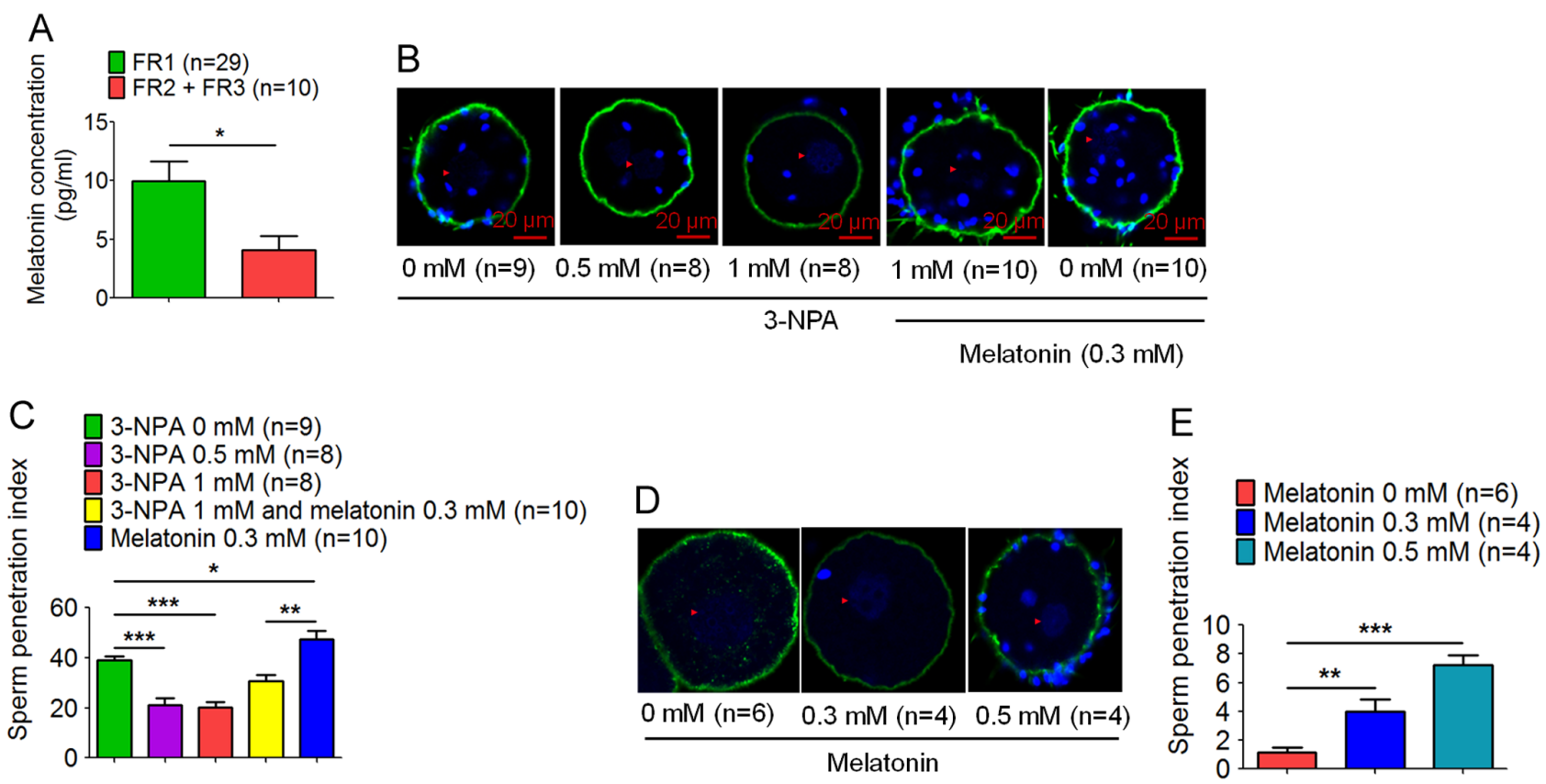

Figure 4 Melatonin rescues the impaired penetration potential of sperm treated with 3-NPA or sperm with poor IVF rate. (A) Melatonin concentration in seminal plasma samples from FR1, FR2 and FR3 groups was assessed by ELISA and shown as bar graph (mean \pm S.E.M.). $P$ values were determined by the Student $t$ test. $n$, number of samples. (B) The penetration of hamster zona-free oocytes by sperm treated without or with 3-NPA $(0.5 \mathrm{mM}$ or $1 \mathrm{mM})$ in the absence or presence of $0.3 \mathrm{mM}$ melatonin were observed by confocal fluorescence microscopy. Green color indicates wheat germ agglutinin (WGA) staining of the membrane of the oocyte. Blue color indicates DAPI staining of the nucleus. The red arrow indicates the nucleus of the oocyte, the other DAPI + nucleus indicate the nucleus of sperm that entered into the oocyte. Scale bar $=20 \mu \mathrm{m}$. (C) The penetration index of sperm treated without or with 3-NPA $(0.5 \mathrm{mM}$ or $1 \mathrm{mM})$ in the absence or presence of $0.3 \mathrm{mM}$ melatonin was shown as bar graph (mean \pm S.E.M.). $P$ values were determined by ANOVA with the Tukey's post hoc test. (D) The penetration of hamster zona-free oocytes by sperm with poor IVF rate in the absence or presence of melatonin $(0.3 \mathrm{mM}$ or $0.5 \mathrm{mM})$ was observed by confocal fluorescence microscopy. Green color indicates WGA staining of the membrane of the oocyte. Blue color indicates DAPI staining of the nucleus. The red arrow indicates the nucleus of the oocyte, the other DAPI+ nucleus indicate the nucleus of sperm that entered the oocyte. (E) The penetration index of sperm with poor IVF rates, in the absence or presence of melatonin $(0.3 \mathrm{mM}$ or $0.5 \mathrm{mM})$ shown as bar graph (mean \pm S.E.M.). $P$ values were determined by ANOVA with the Tukey's post hoc test. *, ** and $* * *, P<0.05, P<0.01$ and $P<0.001$, respectively. $n$, number of oocytes. Data are representive of three independent experiments.

In the present study, we adopted the strategy of hamster, zona-free, oocyte penetration by human sperm to examine whether sperm with poor IVF fertilization rates have reduced penetration ability. Indeed, we found that sperm with poor IVF fertilization rates had significantly attenuated capacity for penetration. Moreover, we found that 3-NPA treatment significantly reduced the penetration ability of human sperm with normal IVF rate. These data suggest that dysfunctional mitochondria in sperm probably lead to unexplained male infertility by impairing the penetration ability of sperm.

Melatonin may increase the activities of mitochondrial complex $\mathrm{I} / \mathrm{IV}$, and further improve cellular respiratory capacity (Jimenez-Aranda et al. 2014). Several other studies demonstrate that melatonin enhances mitochondrial functions through reducing oxidative stress (Manchester et al. 2015, Reiter et al. 2016). The impact of melatonin on sperm and male infertility is still unknown. In the present study, we found that concentrations of melatonin in semen were negatively correlated with IVF rates and less than half of unexplained infertile males from FR2 + FR3 groups as many as that from FR1 group. Melatonin notably reduced mitochondria-derived ROS in sperm with low/poor IVF rates or 3-NPA-treated sperm. Furthermore, melatonin not only improved progressive motility and $\mathrm{ALH}$, but also enhanced the penetration ability into hamster zona-free oocytes of 3-NPA-treated sperm. We also found that melatonin increased the penetration index of sperm with poor IVF rates. Overall, the study supplied evidence that oxidative stress and increased ROS in mitochondria of sperm might lead to IVF failure, but melatonin may improve oxidative stress in mitochondria of sperm as well as the penetration capacity of sperm into oocytes, which further suggests that melatonin might improve IVF outcomes.

In summary, even though the etiology of sperm dysfunction and male infertility is multifactorial and complex, this study provides new insight into male factor infertility during IVF cycles. Mitochondria-derived ROS is an independent biomarker for atypical, male factor infertility, and melatonin has the clinical potential to improve sperm quality. 


\section{Supplementary data}

This is linked to the online version of the paper at https://doi.org/10.1530/REP-19-0231.

\section{Declaration of interest}

The authors declare that there is no conflict of interest that could be perceived as prejudicing the impartiality of the research reported.

\section{Funding}

This work is supported by the National Key Research and Development Program of China (No. 2017YFC1001303 to H-F $\mathrm{H}$ ), the Natural Science Foundation of China (No. 31471405 and 81490742 to H-F H., No. 81270708 and 31671569 to J-Z S), the NSFC-CIHR Joint Health Research Program (No. 8161101434 to H-F H, No. 81361128007 to J-Z S), Key project of the Shanghai Committee of Science and Technology (No. 14DJ1400100 to H-F H).

\section{Author contribution statement}

$\mathrm{H}-\mathrm{F} \mathrm{H}$ had full access to all of the data in the study and takes responsibility for the integrity of the data and the accuracy of the data analysis. $\mathrm{H}-\mathrm{F} \mathrm{H}$ coordinated the study. $\mathrm{H}-\mathrm{F} \mathrm{H}, \mathrm{X}-\mathrm{Y} \mathrm{Z}$, Y-M X co-designed the studies. X-Y Z, Y-M X contributed equally to this study. X-Y Z, Y-M X, L W, R L, L J were responsible for enrolment of participants and arranged informed consent from the participants. X-Y Z, Y-M X, L W, Y-T H, Z-H T, Z-M W, F-H $Y, Z-Q W, Y X$ provided patient care and/or took samples. $X-Y$ Z, Y-M X, Y-J T, X-H L, X-M L, Y Z carried out or supported data analysis, including the statistical analyses. J-Z $S$ helped with the manuscript writing. All authors critically reviewed the report.

\section{Acknowledgements}

Authors thank Dr. Martin Quinn from the United Kingdom for his reading and editing of this manuscript.

\section{References}

Absi E, Ayala A, Machado A \& Parrado J 2000 Protective effect of melatonin against the 1-methyl-4-phenylpyridinium-induced inhibition of complex I of the mitochondrial respiratory chain. Journal of Pineal Research 29 40-47. (https://doi.org/10.1034/j.1600-079X.2000.290106.x)

Agarwal A, Mulgund A, Hamada A \& Chyatte MR 2015 A unique view on male infertility around the globe. Reproductive Biology and Endocrinology 13 37. (https://doi.org/10.1186/s12958-015-0032-1)

Agil A, El-Hammadi M, Jimenez-Aranda A, Tassi M, Abdo W, FernandezVazquez G \& Reiter RJ 2015 Melatonin reduces hepatic mitochondrial dysfunction in diabetic obese rats. Journal of Pineal Research 59 70-79. (https://doi.org/10.1111/jpi.12241)

Amaral A, Lourenco B, Marques M \& Ramalho-Santos J 2013 Mitochondria functionality and sperm quality. Reproduction 146 R163-R174. (https:// doi.org/10.1530/REP-13-0178)

Balaban RS, Nemoto S \& Finkel T 2005 Mitochondria, oxidants, and aging. Cell 120 483-495. (https://doi.org/10.1016/j.cell.2005.02.001)

Barratt CL, Bjorndahl L, Menkveld R \& Mortimer D 2011 ESHRE special interest group for andrology basic semen analysis course: a continued focus on accuracy, quality, efficiency and clinical relevance. Human Reproduction 26 3207-3212. (https://doi.org/10.1093/humrep/der312)

Benz R \& Mclaughlin S 1983 The molecular mechanism of action of the proton ionophore FCCP (carbonylcyanide p-trifluoromethoxyphenylhydrazone). Biophysical Journal 41 381-398. (https://doi.org/10.1016/S00063495(83)84449-X)

Boivin J, Bunting L, Collins JA \& Nygren KG 2007 International estimates of infertility prevalence and treatment-seeking: potential need and demand for infertility medical care. Human Reproduction 221506 1512. (https://doi.org/10.1093/humrep/dem046)

Cheng A, Yang Y, Zhou Y, Maharana C, Lu D, Peng W, Liu Y, Wan R, Marosi K, Misiak M et al. 2016 Mitochondrial SIRT3 mediates adaptive responses of neurons to exercise and metabolic and excitatory challenges. Cell Metabolism 23 128-142. (https://doi.org/10.1016/j.cmet.2015.10.013)

Clyne M 2012 Male factor infertility: effects of ROS and vitamin E on sperm. Nature Reviews Urology 9 62. (https://doi.org/10.1038/nrurol.2011.227)

Cottet-Rousselle C, Ronot X, Leverve X \& Mayol JF 2011 Cytometric assessment of mitochondria using fluorescent probes. Cytometry. Part $A$ 79 405-425. (https://doi.org/10.1002/cyto.a.21061)

Dyer SJ 2009 International estimates on infertility prevalence and treatment seeking: potential need and demand for medical care. Human Reproduction 24 2379-2380; author reply 2380. (https://doi. org/10.1093/humrep/dep219)

Egeberg Palme DL, Rehfeld A, Bang AK, Nikolova KA, Kjærulff S, Petersen MR, Jeppesen JV, Glensbjerg M, Juul A, Skakkebæk NE et al. 2018 Viable acrosome-intact human spermatozoa in the ejaculate as a marker of semen quality and fertility status. Human Reproduction 33 361-371. (https://doi.org/10.1093/humrep/dex380)

Gallon F, Marchetti C, Jouy N \& Marchetti P 2006 The functionality of mitochondria differentiates human spermatozoa with high and low fertilizing capability. Fertility and Sterility 86 1526-1530. (https://doi. org/10.1016/j.fertnstert.2006.03.055)

Gao L, Zhao YC, Liang Y, Lin XH, Tan YJ, Wu DD, Li XZ, Ye BZ, Kong FQ, Sheng JZ et al. 2016 The impaired myocardial ischemic tolerance in adult offspring of diabetic pregnancy is restored by maternal melatonin treatment. Journal of Pineal Research 61 340-352. (https://doi. org/10.1111/jpi.12351)

Gelbaya TA, Potdar N, Jeve YB \& Nardo LG 2014 Definition and epidemiology of unexplained infertility. Obstetrical and Gynecological Survey 69 109-115. (https://doi.org/10.1097/OGX.0000000000000043)

Green DR \& Reed JC 1998 Mitochondria and apoptosis. Science 281 1309-1312. (https://doi.org/10.1126/science.281.5381.1309)

Jimenez-Aranda A, Fernandez-Vazquez G, Mohammad A-Serrano M, Reiter RJ \& Agil A 2014 Melatonin improves mitochondrial function in inguinal white adipose tissue of Zucker diabetic fatty rats. Journal of Pineal Research 57 103-109. (https://doi.org/10.1111/jpi.12147)

Kumar N \& Singh AK 2015 Trends of male factor infertility, an important cause of infertility: a review of literature. Journal of Human Reproductive Sciences 8 191-196. (https://doi.org/10.4103/0974-1208.170370)

Lai B, Zhang L, Dong LY, Zhu YH, Sun FY \& Zheng P 2005 Inhibition of Qi site of mitochondrial complex III with antimycin A decreases persistent and transient sodium currents via reactive oxygen species and protein kinase C in rat hippocampal CA1 cells. Experimental Neurology 194 484-494. (https://doi.org/10.1016/j.expneurol.2005.03.005)

Lapolt PS, Tilly JL, Aihara T, Nishimori K \& Hsueh AJ 1992 Gonadotropininduced up- and down-regulation of ovarian follicle-stimulating hormone $(\mathrm{FSH})$ receptor gene expression in immature rats: effects of pregnant mare's serum gonadotropin, human chorionic gonadotropin, and recombinant FSH. Endocrinology 130 1289-1295. (https://doi. org/10.1210/endo.130.3.1537292)

Li N, Ragheb K, Lawler G, Sturgis J, Rajwa B, Melendez JA \& Robinson JP 2003 Mitochondrial complex I inhibitor rotenone induces apoptosis through enhancing mitochondrial reactive oxygen species production. Journal of Biological Chemistry 278 8516-8525. (https://doi.org/10.1074/ jbc.M210432200)

Lim JA, Li L, Kakhlon O, Myerowitz R \& Raben N 2015 Defects in calcium homeostasis and mitochondria can be reversed in Pompe disease. Autophagy 11 385-402. (https://doi.org/10.1080/15548627.2015.100 9779)

Luo SM \& Sun QY 2013 Autophagy is not involved in the degradation of sperm mitochondria after fertilization in mice. Autophagy 9 2156-2157. (https://doi.org/10.4161/auto.26302) 
Lutjen P, Trounson A, Leeton J, Findlay J, Wood C \& Renou P 1984 The establishment and maintenance of pregnancy using in vitro fertilization and embryo donation in a patient with primary ovarian failure. Nature 307 174-175. (https://doi.org/10.1038/307174a0)

Manchester LC, Coto-Montes A, Boga JA, Andersen LP, Zhou Z, Galano A, Vriend J, Tan DX \& Reiter RJ 2015 Melatonin: an ancient molecule that makes oxygen metabolically tolerable. Journal of Pineal Research $\mathbf{5 9}$ 403-419. (https://doi.org/10.1111/jpi.12267)

Ollero M, Gil-Guzman E, Lopez MC, Sharma RK, Agarwal A, Larson K, Evenson D, Thomas AJ, Jr \& Alvarez JG 2001 Characterization of subsets of human spermatozoa at different stages of maturation: implications in the diagnosis and treatment of male infertility. Human Reproduction $\mathbf{1 6}$ 1912-1921. (https://doi.org/10.1093/humrep/16.9.1912)

Pelgrom LR, Van Der Ham AJ \& Everts B 2016 Analysis of TLR-induced metabolic changes in dendritic cells using the Seahorse XF(e)96 Extracellular Flux Analyzer. Methods in Molecular Biology 1390273 285. (https://doi.org/10.1007/978-1-4939-3335-8_17)

Penefsky HS 1985 Mechanism of inhibition of mitochondrial adenosine triphosphatase by dicyclohexylcarbodiimide and oligomycin: relationship to ATP synthesis. PNAS 82 1589-1593. (https://doi.org/10.1073/ pnas.82.6.1589)

Perez-De La Cruz V, Gonzalez-Cortes C, Pedraza-Chaverri J, Maldonado PD, Andres-Martinez L \& Santamaria A 2006 Protective effect of S-allylcysteine on 3-nitropropionic acid-induced lipid peroxidation and mitochondrial dysfunction in rat brain synaptosomes. Brain Research Bulletin 68379 383. (https://doi.org/10.1016/j.brainresbull.2005.09.013)

Piomboni P, Focarelli R, Stendardi A, Ferramosca A \& Zara V 2012 The role of mitochondria in energy production for human sperm motility. International Journal of Andrology 35 109-124. (https://doi.org/10.1111/ j.1365-2605.2011.01218.x)

Rajender S, Rahul P \& Mahdi AA 2010 Mitochondria, spermatogenesis and male infertility. Mitochondrion 10 419-428. (https://doi.org/10.1016/j. mito.2010.05.015)

Reiter RJ 1980 The pineal and its hormones in the control of reproduction in mammals. Endocrine Reviews 1 109-131. (https://doi.org/10.1210/ edrv-1-2-109)

Reiter RJ, Mayo JC, Tan DX, Sainz RM, Alatorre-Jimenez M \& Qin L 2016 Melatonin as an antioxidant: under promises but over delivers. Journal of Pineal Research 61 253-278. (https://doi.org/10.1111/jpi.12360)

Rosenstock TR, Carvalho AC, Jurkiewicz A, Frussa-Filho R \& Smaili SS 2004 Mitochondrial calcium, oxidative stress and apoptosis in a neurodegenerative disease model induced by 3-nitropropionic acid. Journal of Neurochemistry 88 1220-1228. (https://doi.org/10.1046/ j.1471-4159.2003.02250.x)

Rotterdam ESHRE/ASRM-Sponsored PCOS Consensus Workshop Group 2004 Revised 2003 consensus on diagnostic criteria and long-term health risks related to polycystic ovary syndrome (PCOS). Human Reproduction 19 41-47. (https://doi.org/10.1093/humrep/deh098)

Steer CV, Mills CL, Tan SL, Campbell S \& Edwards RG 1992 The cumulative embryo score: a predictive embryo scoring technique to select the optimal number of embryos to transfer in an in-vitro fertilization and embryo transfer programme. Human Reproduction 7 117-119. (https:// doi.org/10.1093/oxfordjournals.humrep.a137542)

Stefanini M, De Martino C \& Zamboni L 1967 Fixation of ejaculated spermatozoa for electron microscopy. Nature 216 173-174. (https://doi. org/10.1038/216173a0)

Stocco DM 2001 StAR protein and the regulation of steroid hormone biosynthesis. Annual Review of Physiology 63 193-213. (https://doi. org/10.1146/annurev.physiol.63.1.193)

Tocci A \& Lucchini C 2010 WHO reference values for human semen. Human Reproduction Update 16 559; author reply 559. (https://doi. org/10.1093/humupd/dmq019)

Tremellen K 2008 Oxidative stress and male infertility--a clinical perspective. Human Reproduction Update 14 243-258. (https://doi. org/10.1093/humupd/dmn004)

Van Der Windt GJ, Chang CH \& Pearce EL 2016 Measuring bioenergetics in T cells using a Seahorse Extracellular Flux Analyzer. Current Protocols in Immunology 113 16B 1-3-16B 14. (https://doi. org/10.1002/0471142735.im0316bs113)

Van Voorhis BJ 2007 Clinical practice. In vitro fertilization. New England Journal of Medicine 356 379-386. (https://doi.org/10.1056/ NEJMcp065743)

Xu S, Pi H, Zhang L, Zhang N, Li Y, Zhang H, Tang J, Li H, Feng M, Deng P et al. 2016 Melatonin prevents abnormal mitochondrial dynamics resulting from the neurotoxicity of cadmium by blocking calciumdependent translocation of Drp1 to the mitochondria. Journal of Pineal Research 60 291-302. (https://doi.org/10.1111/jpi.12310)

Yamamori T, Yasui H, Yamazumi M, Wada Y, Nakamura Y, Nakamura H \& Inanami $O 2012$ lonizing radiation induces mitochondrial reactive oxygen species production accompanied by upregulation of mitochondrial electron transport chain function and mitochondrial content under control of the cell cycle checkpoint. Free Radical Biology and Medicine 53 260-270. (https://doi.org/10.1016/j.freeradbiomed.2012.04.033)

Yang F, Li L, Chen JP, Liu XQ, Zhong CL, Yang Y, Ren YF, Yuan W, Liang H \& Miao MH 2017 Couple's infertility in relation to male smoking in a Chinese rural area. Asian Journal of Andrology 19 311-315. (https://doi. org/10.4103/1008-682X.168685)

Zhou YS, Webb S, Lettice L, Tardif S, Kilanowski F, Tyrrell C, Macpherson H, Semple F, Tennant P, Baker T et al. 2013 Partial deletion of chromosome 8 beta-defensin cluster confers sperm dysfunction and infertility in male mice. PLOS Genetics 9 e1003826. (https://doi.org/10.1371/journal. pgen.1003826)

Received 27 May 2019

First decision 1 July 2019

Revised manuscript received 2 September 2019

Accepted 10 September 2019 